\title{
Wound repair capacity in type 2 diabetes elder patients: assessment by gene expression profiling (GEP) analysis
}

\author{
M Cuzzola ${ }^{1 *}$, A Dattola ${ }^{1}$, A Meliadò ${ }^{1}$, P Scaramozzino ${ }^{1}$, I Callea ${ }^{1}$, A Pontari ${ }^{1}$, O lacopino ${ }^{1}$, M F Lombardo ${ }^{1,2}$, I Bova ${ }^{1}$, \\ D Marcuccio ${ }^{1}$, R Suraci $^{1}$, G Irrera, P lacopino ${ }^{1}$
}

From de Senectute: Age and Health Forum

Catanzaro, Italy. 5-7 December 2009

\section{Background}

Patients with Type 2 diabetes mellitus (T2DM) have an early endothelial dysfunction associated with poor wound healing rate. Diabetic ulcers often fail to heal, and the mechanism is not well explained because studies of ulcer wounds in humans are limited as a result of the difficulty of obtaining tissue samples. Peripheral blood (PB) can be obtained noninvasively and could potentially overcome this problem.

The aim of present study was to identify T2DM patients at risk of developing poor healing ulcers.

\section{Materials and methods}

As part of our project "Biology and Use of Endothelial Progenitor Cells in Peripheral Occluding Arteriopathy (PAD)", we enrolled a group of newly T2DM patients (26) without vascular complications and ulcers. As positive controls we studied T2DM patients (27) with peripheral arterial occlusive disease (PAD). Normal controls included healthy donors (27).

\section{Results}

We used a TaqMan ${ }^{\circ}$ Low Density Array based on comparative CTdd CT method on Applied Biosystems $7900 \mathrm{HT}$ to perform relative quantification of m-RNA derived from PB samples. Trascriptome included gene products involved in tissue repair events such as fibroblast factors-(FGF 1-2), transforming growth factor- $\beta 1$ (TGF-ß1), collagen type XV and type XVIII and tissue granulin (GR). Inflammation cytokines (TNF, IFN, IL-6, IL-8 IL21) were also investigated.

${ }^{1}$ Centro Unico Regionale Trapianti Cellule Staminali e Terapie Cellulari "Alberto Neri", Azienda Ospedaliera Reggio Calabria, Italy
We found that diabetic subjects showed an imbalance of GEP as compared with normal controls. Altered levels were observed in fibroblastic and collagen transcripts $(p<0,001)$ suggesting that loss of the regenerative potency occurs in diabetic patients even if clinical signs of tissue damage are absent. Inflammation mediators were strongly increased in both groups of diabetic patients, but IL 8 showed a major expression in T2DM subgroup with PAD $(\mathrm{p}<0.000)$.

\section{Conclusions}

We believe that GEP may be a sensitive non-invasive method for the assessment of abnormal repair capacity. Further studies should be carried out, in a larger series, to determine whether patients with diabetes associated with altered GEP should be aggressively treated to reduce the incidence of both micro- and macrovascular complications.

\section{Acknowledgements}

This work was supported by Regione Calabria and Ministero della Salute, Italy.

\section{Author details}

${ }^{1}$ Centro Unico Regionale Trapianti Cellule Staminali e Terapie Cellulari "Alberto Neri", Azienda Ospedaliera Reggio Calabria, Italy. ${ }^{2}$ ARPACal, Vibo Valentia, Italy.

Published: 19 May 2010

doi:10.1186/1471-2318-10-S1-A103

Cite this article as: Cuzzola et al.: Wound repair capacity in type 2

diabetes elder patients: assessment by gene expression profiling (GEP)

analysis. BMC Geriatrics 2010 10(Suppl 1):A103. 